\title{
Spatial Light Modulators: Critical Issues
}

Armand R. Tanguay, Jr.

523 Seaver Science Center

University of Southern California

University Park MC-0483

Los Angeles, California 90089-0483

This special issue of Optical Engineering addresses a number of critical issues in the continuing development and characterization of spatial light modulators for optical processing and computing applications.

Although the last two decades have witnessed tremendous progress in the invention and development of optical processing techniques, architectures, and algorithms, only limited optical hardware is as yet available for the implementation of such concepts in compact systems capable of high frame rate operation. In general, three related bottlenecks exist in optical processors and computers: formatting and storage of the input data field (often for purposes of incoherent-tocoherent and/or serial-to-parallel conversion), space-variant modification of the input image (data field) in either an object or Fourier plane, and space/time-variant detection of the optically processed image. All three require optically or electronically programmable spatial light modulation functions.

A wide variety of candidate spatial light modulators based on diverse physical mechanisms have been proposed, including those that utilize electrooptic, photorefractive, magnetooptic, liquid crystal scattering and reorientation, deformable membrane, acoustooptic, thermoplastic, photochromic, photodichroic, and Franz-Keldysh effects. In the past several years, increased effort has been brought to bear on the critical issues in each spatial light modulator technology that inhibit the full exploitation of each physical mechanism for the optimization of device performance characteristics.

This focus on the fundamental principles of operation of the various spatial light modulator technologies will provide the principal theme of this special issue of Optical Engineering. In particular, it is of great interest to identify those current limitations in device performance characteristics that are of primarily technological, rather than fundamental, origin. To this end, results of device characterization and modeling studies are exceedingly important, especially in so far as they are not only explanatory, but also predictive. It is hoped that the identification of such limitations will be a significant spur to focused research and development in these areas.

The manuscripts that comprise this special issue treat a wide range of spatial light modulators and related materials issues, including devices based on field-modulated total internal reflection (a one-dimensional serial-to-parallel converter), CCD-addressed electrostatically deformable membranes, hybrid field-effect twisted nematic liquid crystal layers (optically addressed, CCD-addressed, and visible-to-infrared converting versions), variable grating mode liquid crystal layers (for implementation of intensity-to-position encoding), microchannel-plate-enhanced charge deposition on electrooptic crystals, and volume holographic storage by means of the photorefractive effect.

Further advances in device and materials technologies for optical processing and computing applications will be the subject of an annual special issue of Optical Engineering, scheduled for publication in the November/December issue in 1984 and in subsequent September issues. Details concerning these issues are provided in the call for papers following the guest editorial. 